\title{
Tracking and Vertexing with the ATLAS Inner Detector in the LHC Run-2
}

\author{
KyungEon Choi, on behalf of the ATLAS collaboration \\ Indiana University, Bloomington IN 47405, USA \\ choiky@indiana.edu
}

\begin{abstract}
Run-2 of the Large Hadron Collider (LHC) has provided new challenges to track and vertex reconstruction with higher centre-of-mass energies and luminosity leading to increasingly high-multiplicity environments, and boosted, highly-collimated physics objects. We present an overview of the ATLAS Inner Detector tracker in Run-2 including the track reconstruction algorithm, time-dependent alignment, tracking and vertexing performance.
\end{abstract}

Keywords: ATLAS Inner detector, tracking, vertexing, Run-2

\section{The ATLAS Inner Detector in Run-2}

The ATLAS Inner Detector (ID), surrounded by a $2 \mathrm{~T}$ solenoid magnet, provides position measurements for charged particles within a pseudorapidity range of $|\eta|<2.5$ [1]. It consists of three subdetectors: Pixel detector, Semiconductor strip Tracker (SCT) and Transition Radiation Tracker (TRT). The Pixel detector consists of 4 barrel layers and 3 end-cap layers, including the Insertable B-Layer (IBL) which has been added as an additional layer to the Pixel barrel system starting from Run-2 [2]. Outside of the Pixel detector, the SCT consists of 4 double-sided barrel and 9 end-cap layers. The TRT is the outermost subdetector of the ID and consists of gaseous proportional straw tubes with electron identification capability using transition radiation photons.

\section{The ATLAS Inner Detector track reconstruction algorithm}

The track reconstruction of the ID consists of several algorithms. For brevity only the main algorithm the inside-out track finding is described [3]. It consists of the following sequence of steps:

- Formation of space-points: The first step is the creation of clusters in the Pixel and SCT, and drift circles in the TRT. Then, clusters and drift circles are transformed into 3D space-points. 
- Track finding seeded by space-points: Sets of three space-points form a seed, either Pixel-only, SCT-only or Mixed seed. Seeds which pass the initial transverse momentum $\left(p_{T}\right)$ and impact parameter resolution cuts are also required to match a fourth space-point that is compatible with the particle's trajectory estimated from the seed. A combinatorial Kalman filter [4] is then used to complete track candidates within silicon detector.

- Ambiguity solving: Track candidates are ranked based on track score, favouring tracks with a higher score. Track candidates are rejected if they fail to meet basic quality criteria. A neural network clustering algorithm has been implemented for the Pixel clusters assigned to multiple track candiates in order to separate multiple particles within a merged cluster in dense environments [5].

- TRT extension: Finally, the track candidates are extended to the TRT if there is a valid set of matching drift circles. Then, a track refit is performed to improve momentum resolution.

\section{Time-dependent alignment}

The ID alignment utilizes the track-based alignment algorithm that minimizes the track-hit residual. For Run-2, the ID alignment framework has been upgraded to correct movements of the ID sub-detectors during data taking runs [6].

The entire Pixel system moves rapidly at the start of a run due to an increased cooling required after turning on the sensor preamplier. In addition, in the IBL a bowing effect is observed within a run, due to an increased power consumption of the front-end electronics with Total-Ionising Dose (TID). The distortion changes as a function of LHC luminosity within a fill.

A time-dependent alignment is implemented to correct these effects and it is fully automated procedure which is done every 20 minutes during first hour of the fill then every 100 minutes after.

\section{Tracking performance}

The intrinsic transverse impact parameter resolution, $\sigma\left(d_{0}\right)$, is below $200 \mu \mathrm{m}$ for low $p_{T}$ tracks and falls below than $20 \mu \mathrm{m}$ for tracks with $p_{T}>10 \mathrm{GeV} / \mathrm{c}$ as shown in the left of Figure 1.

The track reconstruction efficiency is defined as the number of reconstructed tracks matched to truth charged particles divided by the number of truth charged particles in simulation. The track reconstruction efficiency, as shown in the right of Figure 1, reaches up to $90 \%$ for Loose track selections and $80 \%$ for TigHT PRIMARY track selections, which requires more stringent selections to improve a purity [7].

In dense environments, the ATLAS track reconstruction algorithm utilizes a neural network to identify merged pixel clusters and then estimates the track parameters of each cluster [8]. The track reconstruction efficiency is improved for highly energetic jets and $\tau$-leptons which have a comparable separation between 

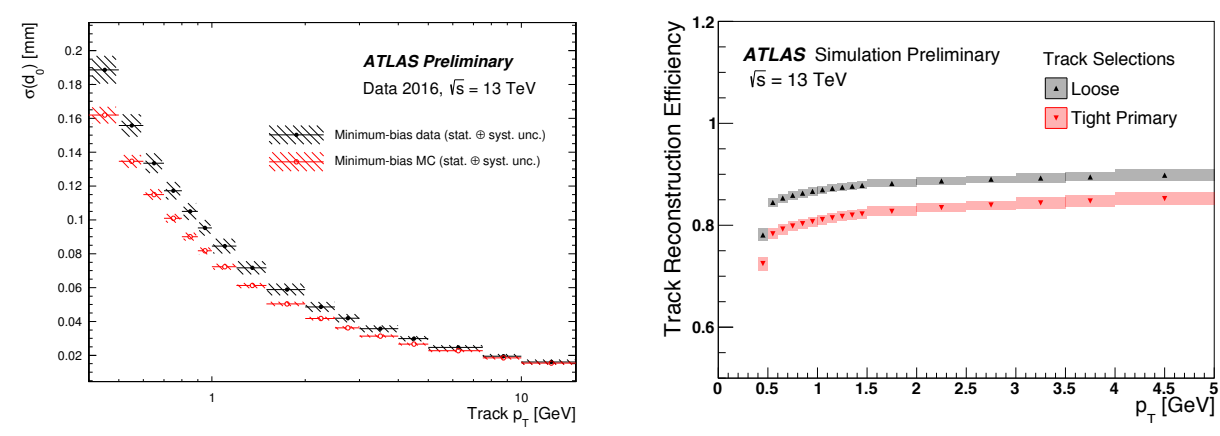

Fig. 1. (Left) the intrinsic transverse impact parameter resolution, $\sigma\left(d_{0}\right)$, is measured in minimum-bias simulation and in 2016 data from proton-proton collisons. (Right) track reconstruction efficiency as a function of truth $p_{T}$ for LOOSE and TIGHT PRIMARY selections of minimum bias simulation.
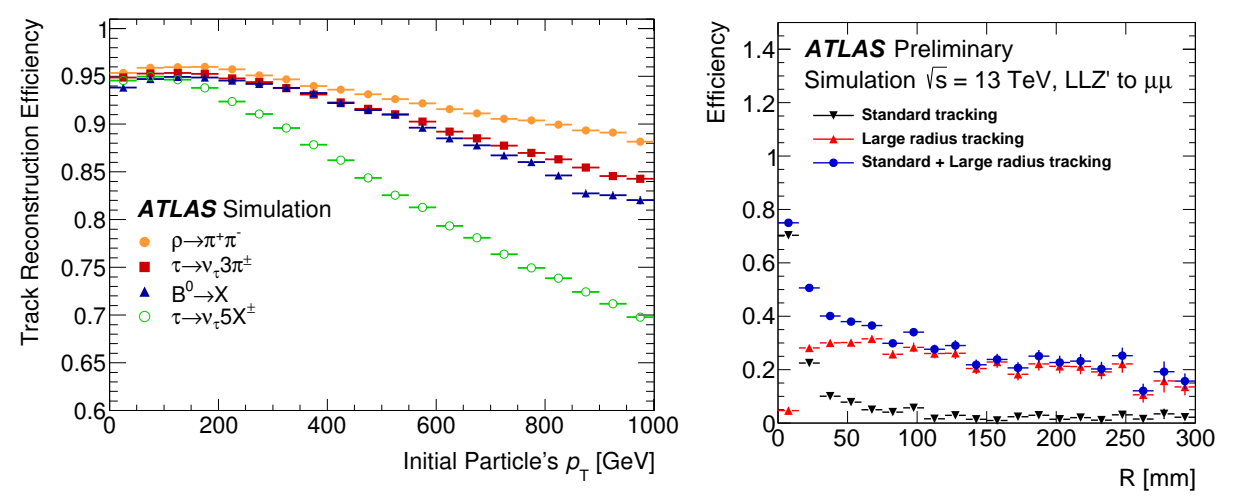

Fig. 2. (Left) track reconstruction efficiency as a function of the initial particle's $p_{T}$ when it is required that the parent particle decays before reaching the IBL for the decay products of a $\rho$, three- and five-prong $\tau$ and $B^{0}$. (Right) track reconstruction efficiency in simulation of long-lived $Z^{\prime}$ bosons as a function of $\mathrm{R}$ the radial distance of the truth production vertex from the origin.

collimated charged particles to the granularity of individual pixels. The track reconstuction efficiency is higher than $80 \%$ for $\rho$, three-prong $\tau$ and $B^{0}$ decays with initial particle's $p_{T}$ of $1 \mathrm{TeV}$ as shown on the left of Figure 2.

The track reconstruction algorithm performs a second pass after the insideout tracking that considers unused hits with tighter $p_{T}$ and looser impact parameter requirements to reconstruct tracks with highly displaced production vertex. Displaced vertices are predicted in several physics beyond the Standard Model scenarios. The track reconstruction efficiency for long-lived $Z^{\prime}$ bosons decaying 
to di-muons is significantly increased by the large radius tracking algorithm as shown on the right of Figure 2.

\section{$5 \quad$ Vertexing performance}

The primary vertex reconstruction utillizes an iterative vertex finding algorithm which progressively down-weights less compatible tracks and then recomputes the vertex position.

The average number of reconstructed vertices is well modeled as a function of the average number of interactions per bunch crossing $(\mu)$ between data and MC. The increase in the average number of reconstructed vertices is smaller with respect to the increase in $\mu$ due to merged vertices and other inefficiencies. Although not all vertices are reconstructed, the vertex reconstruction efficiency of hard-scatter primary vertex is higher than $99 \%$ [9].

The vertex position resolution ranges from 20 to $155 \mu \mathrm{m}$ in $\mathrm{x}$ depending on the number of tracks associated with the vertex without the constraint of the beam-spot [10].

\section{Summary}

The ATLAS Inner Tracking system has had a very successful tracking and vertexing performance and stable operation during the first two years of Run-2.

\section{References}

1. ATLAS Collaboration, The ATLAS Experiment at the CERN Large Hadron Collider, JINST 3 (2008) S08003.

2. ATLAS Colloboration, ATLAS Insertable B-Layer Technical Design Report, CERNLHCC-2010-013.

3. Andreas Salzburger, Optimisation of the ATLAS Track Reconstruction Software for Run-2, Journal of Physics: Conference Series 664 (2015) 072042.

4. R. Frühwirth, Application of Kalman Filtering to Track and Vertex Fitting, Nucl. Instrum. Meth.A 262 (1987) 444.

5. ATLAS Collaboration, A neural network clustering algorithm for the ATLAS silicon pixel detector, JINST 9 (2014) P09009.

6. ATLAS Collaboration, Novel time-dependent alignment of the ATLAS Inner Detector in the LHC Run 2, JINST 11 (2016) C11036.

7. ATLAS Collaboration, Early Inner Detector Tracking Performance in the 2015 Data at $\sqrt{s}=13$ TeV, ATL-PHYS-PUB-2015-051, 2015, URL: https://cds.cern.ch/record/2110140.

8. ATLAS Collaboration, Performance of the ATLAS Track Reconstruction Algorithms in Dense Environments in LHC run 2, (Submitted to Eur. Phys. J. C), arXiv:1704.07983 [hep-ex].

9. ATLAS Collaboration, Reconstruction of primary vertices at the ATLAS experiment in Run 1 proton-proton collisions at the LHC, Eur. Phys. J. C 77 (2017) 332

10. ATLAS Collaboration, Vertex Reconstruction Performance of the ATLAS Detector at $\sqrt{s}=13$ TeV, ATL-PHYS-PUB-2015-026, 2015, URL: https://cds.cern.ch/record/2037717. 